\title{
DELUSIONAL PARASITOSIS: A CASE REPORT
}

R. Fernandes, M. Mendes, M. Melo, D. Pereira, M. Moreno, P. Branco

Centro Hospitalar Psiquiátrico de Lisboa, Lisboa, Portugal

\section{INTRODUCTION}

Delusional parasitosis (DP) or Eckbom syndrome is an uncommon psychiatric disorder, characterized by a fixed false belief of being infested by a parasite. The complaints are usually about skin infestation, but involvement of the gastrointestinal tract has also been described. It can be primary, in which the delusion arises spontaneously as a mono-delusional disorder, or secondary to other psychiatric or organic disease, such as hypothyroidism, cerebrovascular disease, infections, among others.

\section{CASE REPORT}

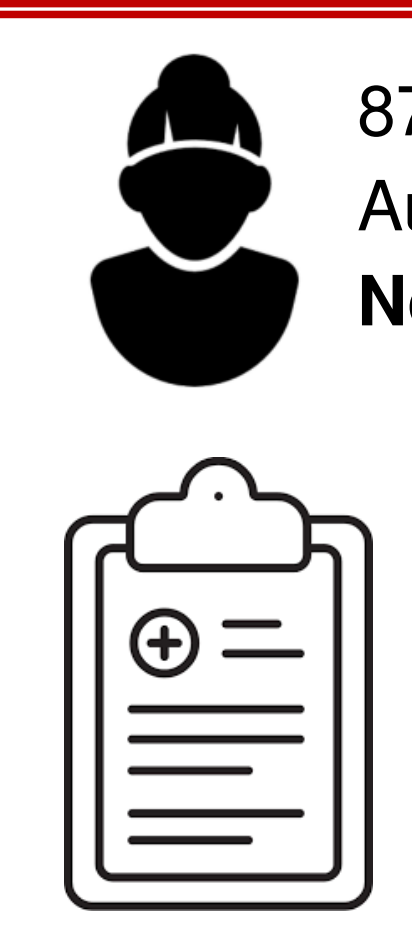

87 year-old woman, widow. Lived alone.

Autonomous in daily living activities.

No previous history of mental ilness.

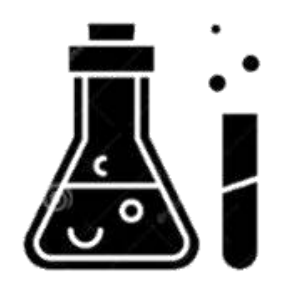

Laboratory exams showed

hypothyroidism (TSH>8; FT4<0,40). TRABs, ATG, ATPO were negative

Referred to the emergency department of psychiatry by the general practitioner presenting the conviction that she was infested by pigeon bugs for approximately ten months. This belief began soon after she decided to foster a pair of pigeons that were living in her balcony. She described them as being "tiny black bugs with wings, 2-3 $\mathrm{mm}$ in length, that released a gelatinous bag full of eggs". She had the sensation that they were digging tunnels under her skin and started pinching her skin, producing serious auto-inflicted lesions on her shoulders. She collected the peeled skin in a jar and claimed that they were bugs. She consulted 8 dermatologists and was given several dermatological treatments with no relief. She held on to her belief regardless of absence of any evidence of an infestation. She was admitted in the psychiatry ward for inpatient management.

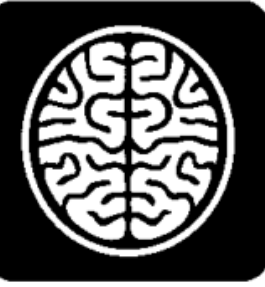

Cranial CT-scan revealed a cortico-subcortical parasagittal anterior left cingulate hypodense lesion (probable vascular etiology) and periventricular white matter hypodensities related to chronic microangiopathic leukoencephalopathy

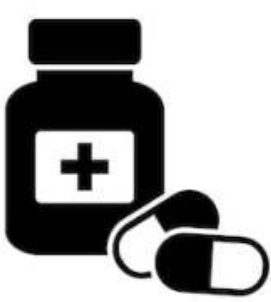

Patient received treatment with thyroid hormone replacement and risperidone $2 \mathrm{mg} /$ day.

\section{She developed medical complications} hyponatremia and hypokalemia- which needed internal medicine collaboration. The treatment was then switched to olanzapine $10 \mathrm{mg} /$ day, with a slow response. The impact of the delusional ideas gradually decreased but they didn't' remit completely.

\section{CONCLUSION}

This is a case of delusional disorder with comorbid organic disorders. Our patient presented some typical described behaviours such as bringing sample of the allegered parasites inside small containers, defined as the "matchbox sign" and providing a detailed description of the "parasites". Similar to most patients with DP she mainly consulted with dermatologists and general practitioners. These patients are often reluctant to see a psychiatrist and to take treatment with antipsychotics because of their belief. For diagnosis and treatment of DP, a close collaboration among dermatologists, psychiatrists, and parasitologists is essential. Patients should be carefully evaluated and there should be a search for organic disorders that are associated. 